\title{
Intracranial Artery Stenting May Not Improve Cognitive Function: A Preliminary Study
}

\author{
Joo Young Kwon, Young Soo Han, Ji Young Kim, Dae Chul Suh, Jae Hong Lee, Dong-Wha Kang, \\ Sun U. Kwon, Jong S. Kim \\ Stroke Center and Department of Neurology, University of Ulsan, Asan Medical Center, Seoul, Korea
}

\section{Dear Sir:}

Although cognitive decline in patients with extracranial atherosclerotic artery stenosis (ECAS) may be improved by carotid revascularization, ${ }^{1}$ the effect of stenting on cognitive dysfunction in patients with intracranial atherosclerosis (ICAS) remains unknown. Although stent placement was not effective in improving overall outcomes of patients with ICAS, the value of stenting may have to be reconsidered if the procedure alleviates cognitive dysfunction. We aimed to evaluate changes in cognitive function and perfusion after stent placement in ICAS patients.

Consecutive patients undergoing stenting who (1) had significant stenosis (>50\%) in the major intracranial arteries and (2) received stenting by consensus of the stroke team (stent group) at the Asan Medical Center, from May 2009 to August 2011, were recruited. Patients who had embolic sources and those with severe cognitive dysfunction who could not undergo neuropsychology (NP) tests were excluded. Control subjects were sex- and age-matched with patients with the same condition, but who refused this procedure for personal reasons.

All patients underwent NP evaluations on the day before the stenting (TO) and at the 12-month (T12) follow-up visit. This included Mini-Mental Status Examination (MMSE), Beck's depression inventory (BDI), and NP tests assessing: (1) attention and executive function-forward/backward digit span test, trail making test $A \& B$ (TMT-A\&B), digit symbol test, and phonemic fluency test; (2) memory-Seoul Verbal Learning Test (SVLT) and Rey Complex Figure Test (RCF), and (3) visuospatial and constructional function-the clock drawing test. The study was approved by our ethical committee, and all the patients provided informed consent. Diamox brain single-photon emission computed tomography (SPECT) was also performed at T0 and T12 in the stent group. $^{3}$

For statistics, the Mann-Whitney U test was used to estimate the differences among continuous variables, and the chi-square and Fisher exact test were used for categorical data. To evaluate the results of NP tests, the differences observed in each patient after 12 months (T12-T0, $\Delta \mathrm{T} 12)$ compared to baseline were calculated. The mean $\Delta$ values were compared between the groups. All statistical analyses were conducted using SPSS for Windows (version 18.0; SPSS Inc., Chicago, IL, USA).

Eleven patients (mean age: 57.3 years, range $48-73$ years) with distal internal carotid artery (ICA) $(n=3)$, middle cerebral artery (MCA) $(n=4)$, basilar artery (BA) $(n=2)$, and distal vertebral artery (VA) $(n=2)$ stenoses and 11 control subjects (mean age: 60.7 years, range $49-72$ years) with MCA $(n=8)$ and BA $(n=3)$ stenoses were enrolled. The stenosis severity of these patients averaged 75\% (range: 50-100). There were no differences in demography, vascular risk factors, the side and degree of stenosis, the length of education, and MMSE, BDI, NIHSS, and NP tests for various domains of cognition between the two groups.

All patients in the stent group underwent successful procedures without any neurological complications, and there was no significant residual stenosis. All patients were followed up for a period of 12 months. Between the two groups, the mean difference between the changes over time in MMSE, BDI, and NP test results for various domains was not significant (Table 1). Initial and follow-up SPECTs were performed in six patients (55\%) from 
Table 1. Changes in cognitive function during the 12-month period, and comparisons of the differences between the stent and control group

\begin{tabular}{|c|c|c|c|c|c|c|c|c|c|}
\hline & \multicolumn{2}{|c|}{ Stent group } & \multicolumn{2}{|c|}{ Control group } & \multicolumn{2}{|c|}{$P$ value $^{+}$} & \multirow{2}{*}{$\begin{array}{c}\text { Stent group } \\
\Delta \mathrm{T} 12\end{array}$} & \multirow{2}{*}{$\frac{\text { Control group }}{\Delta \mathrm{T} 12}$} & \multirow{2}{*}{$P$ value } \\
\hline & T0 & $\mathrm{T} 12$ & T0 & $\mathrm{T} 12$ & T0 & $\mathrm{T} 12$ & & & \\
\hline MMSE & 25.4 & 27.4 & 26.6 & 26.6 & 0.474 & 0.604 & 0.73 & 0 & 0.511 \\
\hline $\mathrm{BDI}$ & 15.9 & 13.3 & 12 & 7.6 & 0.407 & 0.266 & -2.64 & -4.36 & 0.688 \\
\hline \multicolumn{10}{|c|}{ Attention and executive function } \\
\hline Forward digit span & 7.2 & 6 & 7.7 & 6.7 & 0.375 & 0.36 & -1.18 & -1 & 0.776 \\
\hline Backward digit span & 3.5 & 3.8 & 3.9 & 3.7 & 0.492 & 0.892 & 0.27 & -0.18 & 0.245 \\
\hline TMT-A (second)* & 61 & 76.9 & 68.3 & 67 & 0.643 & 0.595 & 4.44 & -1.27 & 0.595 \\
\hline TMT-B (second)* & 149.4 & 163.8 & 143.6 & 151.1 & 0.869 & 0.813 & 5.5 & 2.22 & 0.918 \\
\hline Digit symbol test & 36.9 & 39.6 & 30.2 & 33.6 & 0.392 & 0.528 & 1.5 & 3.45 & 0.607 \\
\hline Phonemic fluency (n) & 12.5 & 12.5 & 11.4 & 11.4 & 0.653 & 0.376 & 0 & -0.91 & 0.644 \\
\hline \multicolumn{10}{|l|}{ Memory } \\
\hline SVLT (total score, n) & 16.6 & 19 & 15.8 & 16.5 & 0.724 & 0.397 & 2.36 & 0.72 & 0.431 \\
\hline SVLT (detailed recall, n) & 4.4 & 4.8 & 4 & 4.6 & 0.772 & 0.901 & 0.73 & 0.64 & 0.927 \\
\hline SVLT (recognition, n) & 18.2 & 19.8 & 19.8 & 18.9 & 0.305 & 0.554 & 0 & -0.91 & 0.723 \\
\hline RCFT (total score) & 24.7 & 31.4 & 30.7 & 31.2 & 0.243 & 0.932 & 0 & 0.5 & 0.821 \\
\hline RCFT (delayed recall) & 9.8 & 4.8 & 12.4 & 4.6 & 0.503 & 0.664 & 5.93 & 3.27 & 0.387 \\
\hline RCFT (recognition) & 18.8 & 19.7 & 20.4 & 19.5 & 0.249 & 0.795 & -1.11 & -0.91 & 0.52 \\
\hline \multicolumn{10}{|c|}{ Visuospatial and constructional function } \\
\hline Clock drawing & 5.8 & 5.4 & 6.7 & 6.2 & 0.137 & 0.439 & -0.45 & -0.55 & 0.917 \\
\hline
\end{tabular}

*Higher values denote more severe impairment, and negative difference score values indicate improvement; ${ }^{\dagger} P$ values are for comparison of T0 and T12 assessments between stent and control patients.

the stent group. Initially, all the patients showed decreased perfusion corresponding to the stenosed artery. The perfusion abnormalities of all six patients improved or normalized after stent placement. However, there was no difference in changes of cognitive function even in these six patients.

The baseline MMSE score in our patients was 26, and the scores from the NP were also generally low, suggesting the presence of diffuse cognitive impairment. This appears to be consistent with a previous study based on transcranial Doppler ultrasonography that reported that low cerebral blood flow velocity was associated with cognitive decline. ${ }^{4}$ We found, however, that stent placement did not affect cognition after 1 year, despite the improved cerebral perfusion in these patients. It seems that there may be brain damages associated with stenting that possibly offset the potential beneficial effects of improved perfusion. ${ }^{5}$ First, the debris of atherosclerotic plaques could be dislodged during the procedure leading to distal embolization. ${ }^{6}$ Generation of microembolic events on transcranial Doppler ultrasonography or new embolic brain lesions detected by diffusion-weighted MRI (DWI) have been reported to occur during or after interventional procedures. ${ }^{7}$ In a previous study, cognitive function tended to decrease more significantly in patients receiving stenting than in those undergoing endarterectomy, which may be related to new ischemic lesions that occur twice as often in the former group. ${ }^{8}$ As only stenting procedures are feasible in treating ICAS, the adverse effect of interventional procedures on cognitive function may be more apparent in these patients.
Second, selective ballooning during the intervention may result in transient cerebral hypoperfusion. In addition, although none of our patients developed overt hyperperfusion syndrome, cerebral hyperperfusion secondary to revascularization may lead to brain damage. ${ }^{9}$ Finally, revascularization of a stenosed intracranial artery may lead to perfusion improvement in smaller areas of the brain than in patients receiving carotid revascularization because the stenosed vessels are located distal to the communicating arteries in ICAS patients.

The merits of our study included our evaluation of the perfusion before and after stenting using Diamox SPECT, which may influence cognitive improvement. Moreover, we performed follow-up NP tests at a 1-year interval to minimize the learning effect. The limitations of our study included the non-randomized design, the lack of SPECT imaging in the control group, and most importantly, the lack of statistical power due to the small number of enrolled patients. Unfortunately, we had to prematurely terminate our study because the number of stenting cases dramatically plummeted after the negative results of the SAMMPRIS study. ${ }^{2}$ However, because we could not see any 'trend' of improvement in stented patients, we suspect that the results may have been the same, even if larger number of patients were enrolled. Despite these limitations, our preliminary results suggest that the stenting procedures may not be of benefit in improving cognitive function in ICAS patients. 


\section{References}

1. Cheng Y, Wang YJ, Yan JC, Zhou R, Zhou HD. Effects of carotid artery stenting on cognitive function in patients with mild cognitive impairment and carotid stenosis. Exp Ther Med 2013;5: 1019-1024.

2. Chimowitz MI, Lynn MJ, Derdeyn CP, Turan TN, Fiorella D, Lane $B F$, et al. Stenting versus aggressive medical therapy for intracranial arterial stenosis. N Engl J Med 2011;365:993-1003.

3. Kim JS, Moon DH, Kim GE, Cho YP, Ryu JS, Lee HK. Acetazolamide stress brain-perfusion SPECT predicts the need for carotid shunting during carotid endarterectomy. J Nucl Med 2000;41: 1836-1841.

4. Ruitenberg A, den Heijer T, Bakker SL, van Swieten JC, Koudstaal PJ, Hofman $A$, et al. Cerebral hypoperfusion and clinical onset of dementia: the Rotterdam study. Ann Neurol 2005;57: 789-794.

5. Goldberg JB, Goodney PP, Kumbhani SR, Roth RM, Powell RJ, Likosky DS. Brain injury after carotid revascularization: outcomes, mechanisms, and opportunities for improvement. Ann Vasc Surg 2011;25:270-286.

6. Vogten JM, Gerritsen WB, Ackerstaff RG, van Dongen EP, de Vries JP. Perioperative microemboli and platelet aggregation in patients undergoing carotid endarterectomy. Vascular 2008;16: 154-160.
7. Bendszus M, Stoll G. Silent cerebral ischaemia: hidden fingerprints of invasive medical procedures. Lancet Neuro/ 2006;5: 364-372.

8. Altinbas $A$, van Zandvoort MJ, van den Berg $E_{1}$ Jongen LM, Algra $A$, Moll $F L$, et al. Cognition after carotid endarterectomy or stenting: a randomized comparison. Neurology 2011;77:10841090.

9. Chida K, Ogasawara K, Suga Y, Saito H, Kobayashi M, Yoshida $K$, et al. Postoperative cortical neural loss associated with cerebral hyperperfusion and cognitive impairment after carotid endarterectomy: 123i-iomazenil SPECT study. Stroke 2009;40: 448-453.

Correspondence: Jong S. Kim

Department of Neurology, Asan Medical Center, University of Ulsan College of Medicine, 88 Olympic-ro 43-gil, Songpa-gu, Seoul 05505, Korea

Tel: +82-2-3010-3442, Fax: +82-2-474-4691

E-mail: jongskim@amc.seoul.kr

Received: November 13, 2015

Revised: December 23, 2015

Accepted: January 7, 2016

This study was supported by a grant from Ministry for Health, Welfare and Family Affairs, Republic of Korea (HI14C1985).

The authors have no financial conflicts of interest. 